left carotid artery endothelium. Another 6 rabbits were fed common diet, no injury. At the beginning and feeding for 12 weeks, TG, TC, HDL, LDL, FBG, fasting insulin and insulin sensitivity index were tested, analysis the correlation between HOMA-IR and lipid. After 12 weeks, OCT images of the left carotid artery were performed. After OCT, specimens were taken. Histology embedded in paraffin and $\mathrm{HE}$ stained was performed on arterial regions that was showed plaques in the OCT scan. OCT results were compared with pathological results.

Results 4 rabbits died, no death in normal. Feeding high fat diet for 12 weeks, their weight, TC, TG, LDL-C, HDL-C were significantly higher than normal $(p<0.01)$. Though the difference of FBG was not statistically significant compared with normal $(p=0.423)$, FSI, ISI and HOMA-IR was statistically significantly different $(\mathrm{p}=0.001$, $0.000,0.000$ respectively). HOMA-IR and TG were positively correlated $(\mathrm{r}=0.52, \mathrm{p}=0.039)$, and TC, HDL, LDL no significant correlation $(p>0.05)$. OCT images of the left carotid artery in 22 rabbits were performed. 60 OCT images analysis after two experts' discussions were held. There were 50 lipid-rich plaques, seven fibrous plaques and three suspected as calcified plaques. In addition, four thrombosis and intima tear in two sites were found. The pathological section was made in corresponding sites to OCT, HE staining. Comparing with the pathology results, OCT had high sensitivity and specificity for atherosclerotic plaque, respectively 96\%, 89\%. Plaque burden, external elastic membrane area and lumens area between the two methods were not statistically different $(p>0.05)$.

Conclusion OCT imaging can clearly visualise different types of atherosclerotic plaques and provide detailed information on plaque characteristics. Comparing with histopathology, OCT had high sensitivity and specificity for characterising atherosclerotic plaque.

\section{e0196 TRB3'-SILENCE REMARKABLY ATTENUATES RENAL FIBROSIS IN DIABETIC RAT}

doi:10.1136/hrt.2010.208967.196

Ding Wenyuan, Ti Yun, Wang Zhihao. Qilu Hospital

Background Renal fibrosis is thought to be the common pathway in most cases of end-stage renal diseases. Recently, it is reported that tribble3 plays an important role in the progress of cardiac fibrosis in diabetes mellitus (DM). Therefore, TRB3 might participate in the pathogenesis of renal fibrosis of diabetes mellitus rat.

Methods 84 male Wistar rats were randomly divided into three groups: control group $(n=12)$, high-fat diet group (HF group, $n=36$ ), DM group $(n=36)$. The rats in DM group were injected with streptozotocin (STZ) after feeding with a high-fat diet for 4 weeks. The last two groups were re-divided into three subgroups according to injection with TRB3 siRNA adenovirus at week 17 whether or not (HF+Ad group; HF+Vector group; HF group; DM+Ad group; $\mathrm{DM}+$ Vector group; DM group; $\mathrm{n}=12$ per group). After rats sacrificed, renal tissue was removed and stained with $\mathrm{H} \& \mathrm{E}$ and masson's trichrosome staining.

Results Renal fibrosis was significantly increased in DM group compared to HF group and Control group $(4.1 \pm 0.87$ vs $0.9 \pm 0.13$ vs $0.53 \pm 0.08 ; p<0.05, p=0.017$, respectively). The severity of fibrosis was also significantly different between three subgroups in DM group, the same to HF group. In DM group, renal fibrosis obviously ameliorate in $\mathrm{DM}+\mathrm{Ad}$ group compared to $\mathrm{DM}+$ Vector group and DM group ( $1.4 \pm 0.24$ vs $7.1 \pm 0.8$ vs $3.7 \pm 0.8 ; \mathrm{p}<0.0001, \mathrm{p}<0.01$, respectively). In HF group, renal fibrosis also noticeably improvement in $\mathrm{HF}+\mathrm{Ad}$ group compared to HF+Vector group and HF group ( $1.3 \pm 0.24$ vs $3.0 \pm 0.8$ vs $0.9 \pm 0.13 ; p<0.05, p<0.05$, respectively).

Conclusion TRB3'silence remarkably attenuates renal fibrosis and gene interference shows beneficial effect in the development of diabetic nephropathy in diabetes mellitus.

\section{e0197 EFFECTS OF PRIOR DIFFERENT INTENSITIES EXERCISE ON INFARCT REGION FUNCTION AND ANGIOGENESIS OF LEFT VENTRICLE IN MYOCARDIAL INFARCTION RATS}

doi:10.1136/hrt.2010.208967.197

${ }^{1}$ Xiang-Yu Tang, ${ }^{2}$ Hua-Shan Hong, 'Liang-Long Chen, ${ }^{1}$ Xiao-Hong Lin, ${ }^{1} J u n-H u a$ Lin, 'Zhang Lin. 'Department of Cardiology of Union Hospital Affiliated to Fujian Medical University; ${ }^{2}$ Department of Geriatrics of Union Hospital Affiliated to Fujian Medical University, Fuzhou, Fujian, China, 350001

Objective Lifestyle interventions including exercise training have been shown to be a feasible option for the prevention and treatment of cardiovascular diseases. In this study, we investigated the effects of prior different intensities of exercise on infarct region and function and angiogenesis of the left ventricle (LV) in post-myocardial infarction (MI) rats, and further examined the expression of VEGF proteins in the left ventricle.

Methods Male Sprague-Dawley rats were randomly assigned to six groups. The exercised rats underwent a daily 60-min treadmill exercise, $5 \mathrm{~d} / \mathrm{wk}$, for $6 \mathrm{wk}$. Different treadmill speeds were carried out in the high intensity exercise group (HIE-MI group, $30 \mathrm{~m} / \mathrm{min}$ ), moderate intensity exercise groups (MIE-Sh and MIE-MI groups, $21 \mathrm{~m} / \mathrm{min}$ ), low intensity exercise group (LIE-MI group, $12 \mathrm{~m} / \mathrm{min}$ ), respectively, whereas the untrained rats (Sed-Sh and Sed-MI groups) remained sedentary. At $6 \mathrm{wk}, 24 \mathrm{~h}$ after the last treadmill exercise or the corresponding sedentary protocol, all rats underwent either acute MI (LIE-MI, MIE-MI, HIE-MI and Sed-MI groups) or sham MI operation (MIE-Sh and Sed-Sh groups). Then, all rats were sacrificed at $7 \mathrm{~d}$ after recovery. Echocardiographic and haemodynamic measurements were performed at the end of the experimental protocol, and hearts were sampled for histological and molecular analysis. The infarct regions were analysed by using Masson's trichrome staining; intramyocardial microvessels were detected by using Factor VIIIrelated antigen staining; and cardiac VEGF protein levels were determined by Western blotting analysis.

Results Compared with Sed-Sh group, MIE-Sh group ameliorated left ventricular function and increased microvessels intensity, though the difference was not significant. Compared with Sed-MI group, MIE-MI and HIE-MI groups significantly reduced left ventricular infarct size, improved haemodynamic parameters, increased fractional shortening, scar thickness and microvessel density, but LIE-MI rats only had slight, and non-significant effect on these parameters. In addition, the differences between MIE-MI and HIE-MI rats were significant in haemodynamic parameters and microvessel density. Interestingly, compared with Sed-MI group, the exercised hearts displayed higher levels of VEGF protein in MIE-MI and HIE-MI groups. However, there was no significant difference between MIE-MI and HIE-MI groups.

Conclusion Moderate intensity running exercise before acute MI improved LV function, reduced scar size, increased scar thickness and microvessel density in the post-MI rat. Additional higher intensity exercise could have little further effect. Low intensity exercise may be beneficial, but not sufficient to improve MI Moderate and high intensity running upregulated the expression of VEGF protein, and contributed to the increased microvessels, which may partly benefit cardiac function after MI.

\section{e0198 ACTIVITY OF CALCINEURIN NFAT SIGNALLING PATHWAY INVOLVING IN REMODELLING IN CTNI R146W MICE}

doi:10.1136/hrt.2010.208967.198

Zhu Shushu, Ma Jizheng, Chen Xiangjian, Wu Hengfang, Yang Di, Zhang Jinan. Nanjing Medical University, China

Objective To construct a transgenic model of HCM overexpressing $\mathrm{cTnI}^{\mathrm{R} 146 \mathrm{~W}}$, observe the pathological change of this animal, and 
elucidate the signalling pathway involving in heart remodelling of cTnI ${ }^{\mathrm{R} 146 \mathrm{~W}+/-}$ mice.

Methods Cardiac hypertrophy-related signalling pathway protein, such as calcineurin, calsarcin-1, GSK-3 $\beta$, AKT, SERCA2, PLB were detected by Western blot and RT-PCR. We also assessed the activity of calcineurin in $\mathrm{cTnI}^{\mathrm{R} 146 \mathrm{~W}+/-}$ mice, in order to elucidate potential mechanisms involving in the cardiac remodelling in $\mathrm{cTnI}^{\mathrm{R} 146 \mathrm{~W}+/-}$ mice.

Results The total expression of $\mathrm{cTnI}$ in $\mathrm{cTnI}^{\mathrm{R} 146 \mathrm{~W}+/-}$ mice was significant higher than $\mathrm{cTnI}^{\mathrm{R} 146 \mathrm{~W}-/-}$ mice $(\mathrm{p}<0.05)$, while the phosphorylation of $c T n I$ decreased significantly $(p<0.05)$, resulting in a obvious decrease of the ratio of phos-cTnI to $c \operatorname{TnI}(p<0.05)$. Pathological changes such as myocardial cell proliferation, cardiac hypertrophy, and interstitial fibrosis were observed by optical microscope in $\mathrm{cTnI}^{\mathrm{R} 146 \mathrm{~W}+/-}$ mice. Markers of cardiac hypertrophy, such as ANF, BNP, $\beta$-MHC increased significantly in $\mathrm{CTnI}^{\mathrm{R} 146 \mathrm{~W}+/-}$ mice $(p<0.05)$. The expression of calsarcin-1 in $\mathrm{cTnI}^{\mathrm{R} 146 \mathrm{~W}+/-}$ mice was significantly higher than that of $c \operatorname{TnI}^{\mathrm{R} 146 \mathrm{~W}-/-}$ mice $(\mathrm{p}<0.01)$, while other cardiac hypertrophy-related signalling pathway protein, such as calcineurin, GSK-3 $\beta$, AKT, SERCA2 did not change. The mRNA expression of PLB was reduced significantly by RT-PCR $(p<0.05)$. Meanwhile, the calcineurin activity of $\mathrm{cTnI}^{\mathrm{R} 146 \mathrm{~W}+/-}$ mice increased significantly $(p<0.01)$.

Conclusion $\mathrm{cTnI}^{\mathrm{R} 146 \mathrm{~W}+/-}$ mice had typical pathological cardiac remodelling and heart dysfunction, especially in the older ones. The expression of calsarcin-1 and the activity of calcineurin-NFAT signalling pathway may be the most important mechanism involving in pathological cardiac hypertrophy in $\mathrm{cTnI}^{\mathrm{R} 146 \mathrm{~W}-1-}$ mice.

\section{e0199 PERSIMIMON PEEL IMPROVED DYSLIPIDEMIA AND ITS RELATED PRODUCTION OF ATHEROGENIC AUTOANTIGEN COMPLEXES IN LOW-DENSITY LIPOPROTEIN RECEPTOR- DEFICIENT MICE}

doi:10.1136/hrt.2010.208967.199

OIAN Nanhu, Yang Zheng. First Affiliated Hospital of Jilin University, Changchun, China

Objective Roles of persimmon peel were investigated on possibility of developing atherosclerosis in low-density lipoprotein receptor (LDLR)-deficient mice in view of lipid metabolism, physico-biological oxidation, production of its related atherogenic autoantigen, and anti-atherogenic natural antibody production.

Method Male LDLR-deficient mice fed a high fat diet or a high fat diet supplemented with $10 \%$ dried and powdered persimmon peel (PP) for 12 weeks.

Result The PP supplementation significantly reduced the increment of plasma cholesterol and triglyceride levels. The high fat diet feeding increased plasma level of oxidised LDL/ $\beta 2$-glycoprotein I (oxLDL/ $/ 2 \mathrm{GPI}$ ) complexes as an atherogenic atuoantigen, while the PP supplementation significantly reduced the increment $(p<0.05)$. After the 12-week feeding, atherosclerotic plaque in the mice with the diet of PP decreased by $70 \%$ as compared to that in mice fed the high fat diet $(\mathrm{p}<0.005)$. The PP feeding also reduced urinary 11dehydrothromboxane $\mathrm{B} 2$, a stable metabolite of platelet activation marker thromboxane A2, but IgM level of anti-oxLDL antibodies was not changed.

Conclusion Thus, these results demonstrate that persimmon peel may have an anti-atherogenic property through normalisation of lipid metabolism and may be able to reduce production of the atherogenic complexes

\section{e0200 EFFECTS OF MESENCHYMAL STEM CELLS ON MATRIX METALLOPROTEINASE SYNTHESIS OF CARDIAC FIBROBLASTS}

doi:10.1136/hrt.2010.208967.200

Ya-Ping Wang, Xin-Yang Hu, Xiao-Jie Xie, Jian-An Wang. Second Affiliated Hospital Zhejiang University College of Medicine, Hangzhou, China

Objectives Mesenchymal stem cell (MSC) transplantation has been known to decrease matrix metalloproteinase (MMP) synthesis in the myocardium after myocardial infarction (MI) and to improve ventricular remodelling. However, the underlying mechanisms behind MSC have not been clearly demonstrated yet. This study investigated the effects of MSCs through paracrine actions on the MMP synthesis of cardiac fibroblasts (CFs).

Methods CFs were placed under hypoxia conditions for $24 \mathrm{~h}$ before co-culture with MSCs or hypoxia preconditioning MSCs (HPMSCs) in transwell. CFs and MSCs/HP-MSCs shared the same medium, in which erythropoietin (EPO) antibody and EPO receptor (EPOR) were/were not added. Gelatin Zymography was used to detect the gelatinolytic activity of matrix metalloproteinase-2 (MMP-2) and matrix metalloproteinase-9 (MMP-9) in culture media of CFs with different conditions. Western-Blotting was used to assay MMP-2, MMP-9 and TIMP-1 synthesis of CFs. The ERK1/2 signalling pathway was also investigated.

Results Protein expression and activity of MMP-2 produced by CFs significantly increased by about 1.4 -fold $(p<0.01)$ through hypoxia and decreased after co-culture with MSCs or H-MSCs. This is not the case with MMP-9. Mediation of effects may involve phosphorylation of ERK1/2. Tissue inhibitors of metalloproteinases-1 (TIMP-1) had reverse effects on regulation of MMP-2. Either exogenous EPOAb or EPOsR partially inhibited MSCs effect on MMP-2 protein expression and activity by CFs.

Conclusions MSCs may influence MMP/TIMP expression by CFs via the ERK1/2 pathway and EPO may acts as a key factor in the paracrine actions of MSCs.

\section{C0201 HEAT SHOCK PROTEIN 90 PROTECTS RAT MESENCHYMAL STEM CELLS AGAINST HYPOXIA AND SERUM DEPRIVATIONINDUCED APOPTOSIS VIA PI3KAKT AND ERK12 PATHWAYS}

doi:10.1136/hrt.2010.208967.201

Gao Feng, Hu Xinyang, Xie Xiaojie, Xu Oiyuan, Wang Yaping, Liu Xianbao, Xiang Meixiang, Sun Yong, Wang Jian-an. Second Affiliated Hospital, Zhejiang University College of Medicine, Hangzhou, China

Objective Mesenchymal stem cells (MSCs) transplantation has shown therapeutic potential to repair the ischaemic and infracted myocardium, but the effects are limited by apoptosis and loss of donor cells in host cardiac microenvironment. The aim of this study is to explore the cytopretection of Hsp90 against hypoxia and serum deprivation induced apoptosis and the possible mechanisms.

Methods Cell viability was determined by 3-(4.5-dimethylthiazol-2yl)-2, 5-diphenyltetrazolium bromide assay. Apoptosis was assessed by Hoechest 33258 nuclear staining and flow cytometric analysis with annexin V/PI staining. The gene expression of TLR4 and ErbB2 was detected by real-time PCR. The protein levels of cleavedcaspase3, bcl-2, bcl-xL, bax, total-Erk, phospho-Erk, total-Akt, phospho-Akt and hsp90 were detected by western-blot. The production of nitric oxide was measured by spectrophotometric assay.

Results Hsp90 improves MSCs viability and protects MSCs against apoptosis induced by serum deprivation and hypoxia. The 